\title{
Vascularização arterial dos lobos torácicos do timo em fetos de suínos da Linhagem $\mathrm{C}^{1}{ }^{1}$
}

\begin{abstract}
Eduardo M.M. Lima ${ }^{2 *}$, Frederico O.C. Silva ${ }^{3}$, Elton, L.S. Rafael ${ }^{3}$, Renato, S. Severino ${ }^{3}$, Sérgio S. Drummond ${ }^{3}$, Pedro P. Bombonato ${ }^{4}$ e Daniela M.Dianese ${ }^{2}$

ABSTRACT.- Lima E.M.M., Silva F.O.C, Rafael E.L.S., Severino R.S., Drummond S.S., Bombonato P.P. \& Dianese D.M. 2009. [Arterial vascularization of the thoracic lobes of the thymus in stillborns of the lineage C40.] Vascularização arterial dos lobos torácicos do timo em fetos de suínos da Linhagem C40. Pesquisa Veterinária Brasileira 29(11):863-867. Faculdade de Agronomia e Medicina Veterinária, Universidade de Brasília, ICC, Ala Sul, Campus Universitário Darcy Ribeiro, Cx. Postal 4508, Brasília, DF 70910-970, Brazil. E-mail: limaemm@unb.br

The origins and distributions of the arteries that vascularization the thoracic lobes of the thymus, right and left, were studied in 30 swine of the lineage C40, fetus, being 12 males and 18 females. The animals had the arterial system filled with aqueous colored solution in a $50 \%$ of Neoprene Latex, and than were fixed in $10 \%$ aqueous solution formaldehyde. The thoracic lobes of thymus were vascularization by direct branches of the right (53.33\%) and left $(63.33 \%)$ internal thoracic arteries, left subclavian (3.33\%), left vertebral (3.33\%), right $(3.33 \%)$ and left $(3.33 \%)$ superficial cervical, left common carotid $(3.33 \%)$, right coronary $(3.33 \%)$ and brachiocephalic trunk (33.33\%) and costocervical trunk (3.33\%). The right internal thoracic arteries (70\%) and left internal thoracic $(76.67 \%)$, left subclavian $(23.33 \%)$, left superficial cervical (3.33\%) of the aortic arch (6.67\%) provided indirect branches for the correspondent thoracic lobes of the thymus in each side.
\end{abstract}

INDEX TERMS: Vascularization, lineage C40, fetus.

RESUMO.- As origens e distribuições das artérias que vascularizaram os lobos torácicos do timo foram estudadas em fetos de 30 suínos da linhagem C40, sendo 12 machos e 18 fêmeas. Os exemplares tiveram o sistema arterial preenchido com solução aquosa a $50 \%$ de Neoprene Látex corado e, em seguida, foram submetidos à fixação em solução aquosa a $10 \%$ de formaldeído. Os lobos torácicos do timo foram vascularizados por ramos diretos das artérias torácica interna direita $(63,33 \%)$ e esquerda $(53,33 \%)$, subclávia esquerda $(3,33 \%)$, vertebral esquerda $(3,33 \%)$,

\footnotetext{
${ }^{1}$ Recebido em 21 de fevereiro de 2008.

Aceito para publicação em 4 de julho de 2009.

2 Faculdade de Agronomia e Medicina Veterinária, Universidade de Brasília, ICC, Ala Sul, Campus Universitário Darcy Ribeiro, Cx. Postal 4508, Brasília, DF 70910-970, Brasil. *Autor para correspondência: limaemm@unb.br

${ }^{3}$ Faculdade de Medicina Veterinária, Universidade de Uberlândia, Campus Umuarama, Av. Pará 1720, Uberlândia, MG 38400-902, Brasil.

${ }^{4}$ Setor de Anatomia dos Animais Domésticos e Silvestres, Departamento de Cirurgia, Faculdade de Medicina Veterinária e Zootecnia, Universidade de São Paulo, Av. Prof. Dr. Orlando Marques de Paiva 87, Cidade Universitária, São Paulo, SP 05508-000, Brasil.
}

cervical superficial direita $(3,33 \%)$ e esquerda $(3,33 \%)$, carótida comum esquerda (3,33\%), coronária direita (3,33\%) e pelos troncos braquiocefálico $(33,33 \%)$ e costocervical $(3,33 \%)$. Observaram-se ainda os ramos indiretos das artérias torácica interna direita $(70 \%)$ e esquerda $(76,67 \%)$, subclávia esquerda $(23,33 \%)$, cervical superficial esquerda $(3,33 \%)$ e do arco aórtico (6,67\%).

TERMOS DE INDEXAÇÃO: Vascularização, linhagem C40, fetos.

\section{INTRODUÇÃO}

A eventual presença de um antígeno no corpo do animal provoca respostas defensivas, de natureza celular e humoral, de responsabilidade dos órgãos linfóides secundários integrantes do organismo. A perfeita gênese destes órgãos se dá com base na pré-existência do órgão linfóide primário, o timo. Por ele se esclarecem os mecanismos de trocas hormonais e celulares dentro dos processos imunológicos (Randall et al. 2000).

O timo apresenta sua importância máxima nos jovens (Dyce et al. 2004), no qual é o maior órgão linfóide, pos- 
suindo uma grande e efetiva atividade linfopoiética (Everett \& Tyler 1967). Assumindo um importante papel para 0 bom desenvolvimento pós-natal do animal e atuando ainda na manutenção da competência imunológica durante toda a vida (Getty 1986 e Dyce et al. 2004).

O melhoramento genético tem sido aplicado intensivamente na melhoria dos suínos explorados industrialmente. O surgimento matrizes suínas geneticamente melhoradas tem sido fator crucial para o sucesso desta cadeia produtiva. Desta forma busca-se o aperfeiçoamento do emprego de matrizes com maior vitalidade, rusticidade e de manejo fácil, para produzirem um maior número de leitões e extrema economia. Para tanto, os suínos da linhagem C40 produzidos pela Dalland do Brasil囚, detém todas as características almejadas, por serem formados por fêmeas livres do gene do estresse e que apresentam alta produção de leite, com consumo adequado de ração, mesmo em climas quentes como do Brasil. Apesar destes aspectos genéticos tão amplamente explorados verifica-se que as particularidades morfofuncionais tem sido pouco ressaltadas. Principalmente ao que tange a morfologia dos órgãos relacionados com os órgãos linfóides, em especial ao timo.

O avanço de técnicas de imunização e o crescente conhecimento nesta área influenciam diretamente o manejo de animais criados em ambientes fechados. Exemplo disso é o que ocorre na suinocultura, onde uma forte medida de controle, através da vacinação, e um bom manejo garantem, em parte, o sucesso da atividade produtiva.

Verifica-se assim que o timo acaba ocupando o ápice desta cadeia ao fornecer subsídios biológicos para o perfeito desenvolvimento animal. No entanto, sua morfologia e suas funções não estão totalmente esclarecidas. Desta forma a presente investigação científica visa conhecer os dados relativos à vascularização arterial dos lobos torácicos do timo em fetos de suínos C40 estabelecendo para tanto os aspectos morfológicos pertinentes à análise do número, origem e ordenação dos vasos arteriais destinados a este órgão.

\section{MATERIAL E MÉTODOS}

Para este trabalho foram utilizados 30 fetos de suínos da linhagem C40, sendo 18 fêmeas e 12 machos. O material foi originado de abortos e mortes naturais de fêmeas gestantes de núcleos criatórios da região de Uberlândia, MG. Após a coleta o sistema arterial foi preenchido com solução aquosa a $50 \%$ de Neoprene Látex "450", corada com pigmento específico. Para a injeção desta solução no sistema arterial, procedeu-se a identificação, isolamento e a canulação da aorta descendente.

Posteriormente, os fetos foram fixados em solução aquosa a $10 \%$ de formaldeído, mediante injeções subcutâneas e intramusculares e ainda por imersão. A dissecação dos lobos torácicos do timo, direito e esquerdo, deu-se após a remoção das paredes do tórax de ambos os antímeros, mediante secções das articulações costotransversais e costocondrais. Foi empregado ainda campo visual de uma lupa monocular do tipo Wild (10x).
Os termos estiveram de acordo com o preconizado pela Nomina Anatômica Veterinária (2005). Os achados foram registrados de acordo com a origem, o número e ordenação das artérias que supriram os lobos torácicos do timo, em ambos os antímeros. Estes foram submetidos a tratamento estatístico a partir da aplicação do teste de Wilcoxon (Siegel, 1975), com nível de significância de 5\%.

\section{RESULTADOS}

Em relação a presença dos lobos torácicos do timo, foi possível observar que o lobo torácico do timo esquerdo esteve presente em todos os casos analisados (100\%). De outra forma o lobo torácico direito do timo esteve ausente em dois animais (6,67\%), em outros seis animais $(20 \%)$ o lobo torácico direito mostrou-se reduzido de tamanho, em comparação ao seu par, no antímero esquerdo. Já a presença do istmo cervicotorácico do timo foi verificado em 24 dos espécimes (80\%).

Os lobos torácicos do timo direito e esquerdo foram supridos por ramos das artérias torácicas internas direita e esquerda, subclávia esquerda, tronco braquiocefálico, cervicais superficiais direita e esquerda, carótida comum esquerda, arco aórtico, tronco tireocervical esquerdo, vertebral esquerda e coronária direita (Quadros 1 e 2).

Independente da origem, do tipo e das associações ocorridas, o número variável de colaterais que penetraram os lobos torácicos do timo variaram de dois a oito, sendo que cinco ramos em dez animais (30\%), quatro e sete ramos em cinco animais (16,67\%), três e seis ramos em quatro animais $(13,33 \%)$, oito ramos em dois animais $(6,67 \%)$ e dois ramos em um animal (3,33\%).

O lobo torácico direito do timo este foi vascularizado por ramos diretos da artéria torácica interna direita que cedeu um ramo em quinze casos (50\%) e três ramos em

Quadro 1. Número de ramos diretos das artérias que vascularizaram o lobo esquerdo do timo em fetos de suínos da linhagem C40

\begin{tabular}{lcccc}
\hline \multirow{2}{*}{ Artérias } & \multicolumn{4}{c}{ Número de ramos diretos } \\
\cline { 2 - 5 } & 1 & 2 & 3 & 4 \\
\hline Subclávia esquerda & $3,33 \%$ & - & - & - \\
Tóracica interna esquerda & $33,33 \%$ & $13,33 \%$ & $13,33 \%$ & $3,33 \%$ \\
Vertebral esquerda & $3,33 \%$ & - & - & - \\
Cervical superficial esquerda & $3,33 \%$ & - & - & - \\
Tronco braquiocefálico & $33,33 \%$ & - & - & - \\
Carótida comum esquerda & $3,33 \%$ & - & - & - \\
Coronária direita & $3,33 \%$ & - & - & - \\
Tronco costocervical & $3,33 \%$ & - & - & -
\end{tabular}

Quadro 2. Número de ramos indiretos das artérias que vascularizam o lobo esquerdo do timo em fetos de suínos da linhagem C40

\begin{tabular}{lccccc}
\hline \multirow{2}{*}{ Artérias } & \multicolumn{5}{c}{ Número de ramos diretos } \\
\cline { 2 - 6 } & 1 & 2 & 3 & 4 \\
\hline Subclávia esquerda & $20 \%$ & - & $3,33 \%$ & - & - \\
Torácica interna esquerda & $43,33 \%$ & $20 \%$ & $6,67 \%$ & $3,33 \%$ & $3,33 \%$ \\
Cervical superficial esquerda & $3,33 \%$ & - & - & - & - \\
Arco aórtico & $3,33 \%$ & $3,33 \%$ & - & - & -
\end{tabular}




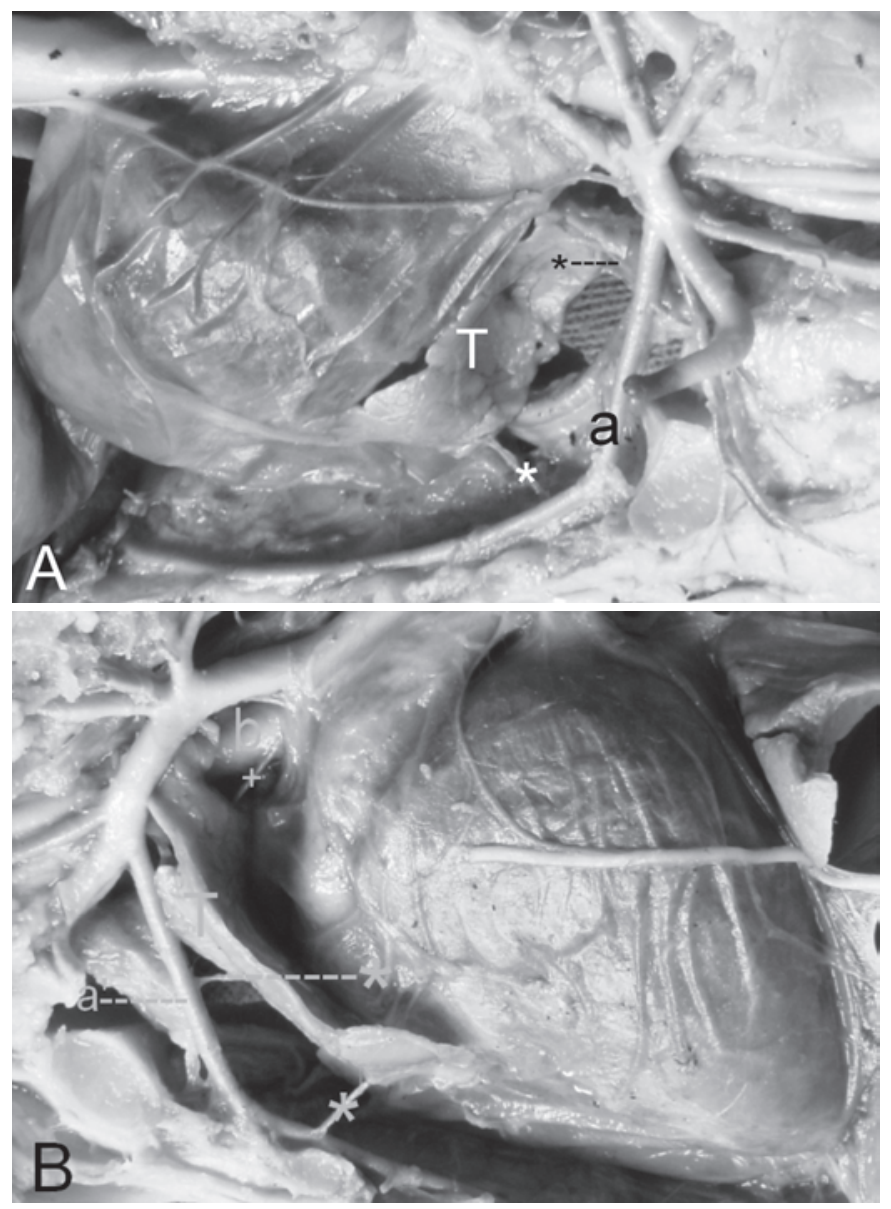

Fig.1. Cavidades torácicas de fetos de suínos da linhagem C40, (A) nos antímeros direito e (B) esquerdo. Lobos torácicos do timo $(\mathrm{T})$ correspondentes, recebendo ramos diretos $\left({ }^{*}\right)$ das artérias torácica interna direita (a) e esquerda (b) e ainda ramos diretos (+) da artéria carótida comum esquerda (b).

um caso (3,33\%) (Fig.1). Este lobo recebeu ainda da artéria cervical superficial direita um ramo direto em um caso (3,33\%). Quanto ao número de ramos indiretos destinados ao lobo torácico direito do timo, a artéria torácica interna direita forneceu um ramo em doze casos (40\%) e dois ramos em nove casos (30\%).

Ao instante da aplicação do teste de Wilcoxon (Siegel 1975), com nível de significância estabelecido em 0,05 em uma prova bilateral, foram encontradas diferenças significantes entre o número de ramos indiretos e também o de ramos diretos que vascularizaram o lobo torácico esquerdo do timo, considerando-se todos os animais e ainda em relação ao sexo dos mesmos.

\section{DISCUSSÃO}

A divisão entre os lobos cervical e torácico do timo no presente estudo esteve de acordo com o estabelecido por Drummond et al. (1997), Silva et al. (1999, 2003 e 2007) e NAV (2005), quando tal divisão ocorreu ao nível da borda cranial do primeiro par de costelas.
Para Bombonato et al. (2001), em fetos de caprinos da raça Saanen, o istmo cervicotorácico foi observado em $61,11 \%$ das peças, ocupando a margem caudal do primeiro par de costelas até a projeção dorsal correspondente à sétima vértebra cervical. Já nos suínos da linhagem C40 o istmo cervicotorácico esteve presente em $80 \%$ dos casos.

Especificamente em suínos, a vascularização do timo de suíno foi realizada em Hampshire (Drummond et al. 1997), sem raça definida (Drummond et al. 1996), Hampshire (Gonçalez et al. 2003) e em suínos da raça Piau (Silva et al. 2003). Nos estudos realizados na raça Hampshire, Drummond et al. (1997) comentaram que a massa tímica não foi encontrada no antímero direito em $26,67 \%$ destes animais. De outra forma, Drummond et al. (1996) citou que os lobos torácicos do timo estiveram completamente ausentes em 3,33\% dos suínos sem raça definida. Para Gonçalez et al. (2003) esta ausência ocorreu em $30 \%$ dos suínos da raça Hampshire e nos achados de Silva et al. (2003) em $10 \%$ dos suínos da raça Piau. Indo de encontro com estes pesquisadores verificou-se que em $6,67 \%$ dos suínos da linhagem C40 o lobo torácico direito do timo não foi evidenciado. De outra forma, o lobo torácico direito do timo mostrou-se de tamanho reduzido em seis animais (20\%). Sobretudo em todos os fetos deste estudo o lobo torácico esquerdo do timo mostrou-se proporcionalmente mais desenvolvido que o direito, indo ao encontro assim com os achados de Gonçalez et al. (2000).

No tocante ao suprimento arterial sanguíneo para o lobo torácico do timo verificou-se que o lobo torácico esquerdo do timo recebeu um total de 105 ramos arteriais, 54 indiretos e 51 diretos, contra apenas 49 ramos enviados para o lobo torácico direito, sendo 30 ramos indiretos e 19 diretos. Isto vem de encontro com o fato da sintopia que ocorreu entre os vasos arteriais que se mostraram dispostos no antímero esquerdo da cavidade torácica e o lobo torácico do timo correspondente. Tal comportamento foi estatisticamente comprovado. Ao instante da aplicação do teste Wilcoxon, com nível de significância de 0,05 , que verificou diferenças significativas entre o número de ramos diretos e os indiretos, considerando-se todos os animais, sendo que os valores mais elevados em ambos foram aqueles relativos ao lobo torácico esquerdo do timo.

Quando analisados os informes de Getty (1986), Silva et al. (1993, 1994, 1999, 2003), Drummond et al. (1996, 1997, 2000), Machado et al. (1999) e Gonçalez et al. (2000, 2003) verificaram-se semelhanças relativamente às fontes de vascularização destinadas aos lobos tímicos, ao instante da participação das artérias torácicas internas direita e esquerda, subclávias direita e esquerda e ainda o tronco braquiocefálico. Assim, coincidindo com o observado em suínos da linhagem C40, com exceção da participação nestes animais da artéria subclávia direita.

De acordo com Schummer et al. (1981) os lobos torácicos do timo relacionaram-se com o pericárdio e com o esterno, este arranjo favoreceu assim a participação das artérias dispostas mais próximas ao eixo percorrido 
pelo esterno contribuírem com a vascularização do timo. Assim entende-se a relação sintópica das artérias torácicas internas direita e esquerda na participação do suprimento sanguíneo para os lobos torácicos do timo em fetos de suínos da linhagem C40. Fato que coincidiu assim com os relatos de Silva et al. (1999, 2003, 2007), Gonçalez et al. (2003) e Dyce et al. (2004) e quando a artéria torácica interna cedeu ramos colaterais ramos para o timo.

Drummond et al. (1997) salientou que o lobo torácico do timo esteve disposto em posição dorso ventral no mediastino cranial, ocupando o antímero esquerdo. $O$ fato da participação do tronco braquiocefálico suprindo somente o lobo torácico esquerdo pode ser explicado também neste deslocamento do mediastino cranial para o antímero esquerdo, onde o timo pôde se desenvolver. Pois a projeção dorsal do órgão neste espaço possibilitou íntima relação com este vaso.

O deslocamento do timo no sentido dorsal permitiu também a íntima relação deste com a aorta e a proximidade com as artérias vertebrais, tronco costocervical, cervical superficial direita e esquerda, tronco bicarótico entre outras, como as artérias subclávias direita e esquerda. Assim, estes vasos foram observados por Getty (1986), Drummond et al. (1997, 2000), Gonçalez et al. (2000, 2003), Dyce et al. (2004) e Silva et al. (2007) e também encontrados nos fetos de suínos da linhagem C40.

No tocante a artéria subclávia esquerda pode-se ver sua participação na vascularização do lobo torácico do timo, especificamente o esquerdo, enviando ramos diretos $(3,33 \%)$ e indiretos $(23,33 \%)$. O maior número de ramos indiretos provindos por esta artéria deve-se pela diversidade de estruturas supridas. Também, em razão do deslocamento do mediastino cranial para o antímero esquerdo e o maior desenvolvimento tímico neste antímero, observa-se somente a contribuição a partir da artéria subclávia esquerda.

Haja vista as características sintópicas, os relatos da participação das artérias cervical superficial direita e esquerda podem ser vistos pelos informes prestados por Silva et al. (1993, 1994), Drummond et al. (1996) e Gonçalez et al. (2000, 2003); das artérias pericardicofrênicas por Silva et al. (1999, 2003); das artérias carótidas comuns direita e esquerda por Silva et al. (1993, 1994), Gonçalez et al. (2000, 2003); do tronco bicarótico por Silva et al. $(1999,2003)$ e vertebral esquerda por Silva et al. (2003). Diante destes informes verificou-se que nos espécimes estudados a participação destas artérias acrescentando-se ainda o tronco costocervical esquerdo, o arco aórtico e a artéria coronária direita.

Referindo-se especificamente com as artérias coronárias, Ehrich et al. (1931) comentaram a respeito da circulação coronária bilateralmente balanceada e do seu grau de variação, salientaram que a artéria coronária esquerda tem em média, apenas $2 / 3$ do número de ramos da artéria contralateral. Esta diferença é compensada porque $1 / 4$ dos ramos da artéria coronária esquerda dirigiram-se no sentido do ápice do coração, disposição que constituiu argumento a favor de igual desenvolvimento das duas artérias coronárias. Esta maior quantidade de ramos originários da artéria coronária direita, em comparação com a artéria contralateral, ao nível da base do coração, aumentaram as chances da mesma contribuir com a vascularização do lobo torácico do timo, principalmente quando o mesmo avançou no sentido dorsal do mediastino cranial. O que de fato foi evidenciado em nossos estudos, quando observou a artéria coronária direita contribuindo para a vascularização do lobo torácico esquerdo do timo.

\section{CONCLUSÕES}

Com base nos resultados da presente investigação científica pode-se concluir que em fetos de suínos da linhagem C40:

- O sexo dos animais não é uma covariável com influência na freqüência e nem no tipo de ramos arteriais que vascularizaram cada lobo torácico do timo;

- Os lobos torácicos direito e esquerdo do timo foram supridos por ramos das artérias torácicas internas direita e esquerda, subclávia esquerda, tronco braquiocefálico, cervicais superficiais direita e esquerda, carótida comum esquerda, arco aórtico, tronco costocervical esquerdo, vertebral esquerda e coronária direita;

- As artérias torácicas internas direita e esquerda foram as principais responsáveis pela vascularização dos lobos torácicos do timo, em ambos os antímeros;

- Os ramos arteriais endereçados aos lobos torácicos do timo, relativamente ao número, origem e ordenação esteve disposto em sentido crânio caudal, o que indicou também a existência de um arranjo peculiar para cada indivíduo;

- O número de ramos indiretos e diretos que vascularizaram o lobo torácico esquerdo do timo são diferentes entre si.

\section{REFERÊNCIAS}

Bombonato P.P., Santana M.I.S. Silva F.O.C., Severino R.S. \& Drummond S.S. 2001. Irrigação do timo de fetos caprinos da raça Sannen: estudo comparativo. Braz. J. Vet. Res. Anim. Sci. 38(5):203-208.

Drummond S.S., Bombonato P.P., Silva F.O.C., Severino R.S. \& Santos A.L.Q. 1996. Vascularização arterial do timo em suínos s.r.d. Braz. J. Vet. Res. Anim. Sci. 33(4):214-219.

Drummond S.S., Bombonato P.P., Silva F.O.C. \& Severino R.S. 1997. Aspectos morfométricos e vascularização arterial do timo em suínos da raça Hampshire. Braz. J. Vet. Res. Anim. Sci. 34:173-178.

Drummond S.S., Silva F.O.C., Severino R.S., Martins A.K., Cardoso J.R., Okuda H.T. \& Gonçales E.M. 2000. Irrigação da porção torácica do timo de fetos e recém nascidos de suínos da raça Moura. Vet. Notícias 6:13-17.

Dyce K.M., Sack W.O. \& Wensing C.J.G. 2004. Tratado de anatomia veterinária. 3를 ed. Elsevier, Rio de Janeiro. 813p.

Ehrich W., de la Chapelle C. \& Cohn A.E. 1931. Anatomical ontogeny of man: A study of the coronary arteries. Am. J. Anat. 49:241-282.

Everett N.B. \& Tyler R.N. 1967. Lymphopoiesis in the thymus and other tissues: Functional implication. Int. Ver. Cytol. 22:205-237. 
Getty R. 1986. Sisson/Grossman Anatomia dos Animais Domésticos. 5a ed. Guanabara Koogan, Rio de Janeiro, p.258-1273.

Gonçalez P.O., Silva F.O.C., Severino R.S., Drummond S.S., Bombonato P.P. \& Machado G.V. 2000. Suprimento arterial da parte torácica do timo em fetos de suínos da Raça Pietrain. Vet. Notícias 6:19-24.

Gonçalez P.O., Silva F.O.C., Severino R.S. \& Drummond S.S. 2003. Suprimento arterial do lobo torácico do timo em suínos da linhagem "Rezende". Ciência Rural 33(3):501-505.

International Committe on Veterinary Gross Anatomical Nomenclature. 2005. Nomina Anatomica Veterinária. 5th ed. Hannover, Alemanha.

Machado G.V., Silva F.O.C., Severino R.S., Drummond S.S., Santos A.L.Q., Bombonato P.P. \& Nascimento K.N. 1999. Suprimento arterial do timo em suínos da raça Duroc. Arq. Ciênc. Vet. Zool. Unipar 2(1):17-22.

Randall D., Burggren W. \& French K. 2000. Fisiologia Animal: mecanismos e adaptações. $4^{\underline{a}}$ ed. Guanabara Koogan, Rio de Janeiro. $445 p$.

Schummer A., Wilkens H., Vollmerhaus B. \& Habermehl K.H. 1981. The Anatomy of the Domestic Animals. Paul Parey, Berlin, p.283288.
Siegel S. 1975. Estatística Não-Paramétrica, para as Ciências do Comportamento. McGraw-Hill do Brasil, São Paulo. 350p.

Silva F.O.C., Severino R.S., Santos A.L.Q., Drummond S.S., Bombonato P.P. \& Nascimento K.N. 1993. Suprimento arterial do timo em fetos de suínos da raça Landrace. 11aㅗ Semana Científica de Medicina Veterinária, Uberlândia, p.252-253.

Silva F.O.C., Bombonato P.P., Severino R.S., Drummond S.S., Santos A.L.Q. \& Nascimento K.N. 1994. Suprimento arterial do timo em fetos de suínos da raça Large White. 23ํㅡㄹ Congresso Brasileiro de Medicina Veterinária, Olinda, p.15.

Silva F.O.C., Machado G.V., Severino R.S., Drummond S.S., Santos A.L.Q., Bombonato P.P. \& Rezende R.J. 1999. Suprimento arterial para o lobo torácico do timo em fetos de suínos SRD. Arq. Ciênc. Saúde Unipar 3(1):3-8.

Silva F.O.C., Severino R.S., Drummond S.S., Bombonato P.P., Bento L.R.T. \& Lima E.M.M. 2003. Artérias dos lobos torácicos do timo em Sus scrofa da raça Piau. Biotemas 16(1):109-119.

Silva F.O.C., Rafael E.L.S., Severino R.S., Drummond S.S. \& Bombonato P.P. 2007. Vascularização arterial dos lobos cervicais do timo em fetos de suínos da linhagem C40. Pesq. Vet. Bras. 27(6):246-250. 